\title{
Multi Model Criteria for the Estimation of Road Traffic Congestion from Traffic Flow Information Based on Fuzzy Logic
}

\author{
Hari Shankar, P. L. N. Raju, K. Ram Mohan Rao \\ Geoinformatics Division, Indian Institute of Remote Sensing, Dehradun, India \\ Email: rammohan@iirs.gov.in
}

Received September 26, 2011; revised October 28, 2011; accepted November 20, 2011

\begin{abstract}
In this study, the road traffic congestion of Dehradun city is evaluated from traffic flow information using fuzzy techniques. Three different approaches namely Sugeno, Mamdani models which are manually tuned techniques, and an Adaptive Neuo-Fuzzy Inference System (ANFIS) which an automated model decides the ranges and parameters of the membership functions using grid partition technique, based on fuzzy logic. The systems are designed to human's feelings on inputs and output levels. There are three levels of each input namely high, medium and low for input density, fast, medium and slow for input speed, and five levels of output namely free flow, slow moving, mild congestion, heavy congestion and serious jam for the road traffic congestion estimation. The results, obtained by fuzzy based techniques show that the manually tuned Sugeno type technique achieves $72.05 \%$ accuracy, Mamdani type technique achieves 83.82\% accuracy, and Adaptive Neuro-Fuzzy Inference System technique achieves $88.23 \%$ accuracy. ANFIS technique appears better than the manually tuned fuzzy technique, and also the manually tuned fuzzy technique gives good accuracy which leads that the fuzzy inference system can capture the human perception better through manual adjustment of input/output membership functions.
\end{abstract}

Keywords: Fuzzy Inference Systems; Fuzzy Rules; Congestion; Human Perception

\section{Introduction}

In general, the road traffic congestion of urban transport is defined as the ratio of volume to the capacity of the road $[1,2]$. However, the volume and capacity (demand) of the road are not directly measurable quantities and thus the value of congestion becomes subjective in nature. Hence, in this paper the directly and precisely measurable quantities such as density and speed of the vehicles, are used for the estimation of road traffic congestion [3]. These two traffic parameters are considered in this paper by keeping in mind that general perception about the congestion on the roads increases when the number of traffic (traffic density) increases and also increases when the speed of the traffic decreases. By the same terminology the road traffic congestion can be defined as the ratio of density to the speed of the vehicles. These two parameters are the inputs to the fuzzy model, and the output of the model is level of congestion. In fact, the actual traffic conditions are not perfectly matches with the conventional way of determining the congestion level using volume and capacity, because there are a number of other factors which affect the congestion and also depends upon the human feelings [4]. But using these directly measurable quantities, the subjectivity of the conventional method of determining congestion level using volume to capacity ratio is removed.

The road traffic congestion is one of the most confusing tasks, because there is no standard way of measuring congestion level on the roads and intersections. It results in serious environmental, time wastage, health hazards, and economic problems. Thus, it is very important to detect where the congestion occurs, as well as to measure and estimate how the congestion is. There may be a number of solutions of road traffic congestion like road pricing, fuel levies, expansion and improvisation of railway lines, and elimination of roundabouts. The main objective of this study is to estimate the road traffic congestion using fuzzy techniques. Therefore, the fuzzy techniques are used to tackle this problem by using the traffic flow information such as speed and density of the vehicles. The fuzzy logic is well known to be suitable for handling problems that are nonlinear in nature such as human feelings $[5,6]$. Road congestion is a subjective quantity, because it comes from the feelings of vehicle driver and decision makers which may be different for different drivers or decision makers. In the same road 
conditions, some may feel that the road is heavily congested, while some others may feel that the road is only slightly congested. This is the problem of mismatching data interpretation due to different user's perception. In traveler navigation system, publication of congestion degree will provide drivers useful information, thus, reduce traffic jam, increase efficiency of trips, and avoid wastage of fuel consumption. It is well known that the process from free flow to serious jam is continuous. It can be represented by a continuous number, say level of congestion (LOC). The LOC is related to the basic traffic parameters such as speed and density. There are other traffic parameters also which affects the congestion level but in this study we use only two traffic parameters i.e. speed and density, which affect mostly to congestion. The objective of this study is to estimate the level of congestion of a road segment using different fuzzy models namely Sugeno-type Fuzzy Inference System, Mamdani-type Fuzzy Inference System and Adaptive NeuroFuzzy Inference System [7], to evaluate the performance of fuzzy inference systems by measuring accuracy of system outputs against human opinion.

\section{Study Area}

Dehradun city is chosen as the study area for assessing the traffic congestion. A road segment with two lanes from Inter State Bus Terminal Dehradun to Saharanpur is taken as a test case to quantify the road traffic congestion. Figure 1 shows the study area depicting the road network of Dehradun city. This road segment has two lanes, going from ISBT Dehradun to Saharanpur, near ISBT Dehradun Uttarakhand. In this research framework, five levels of congestion are defined for traffic congestion, namely free flow, slow moving, mild congestion, heavy congestion and serious jam, and estimating them by using only traffic video.

\section{Literature Review}

Porikli and Li, 2004 determine five level of congestion from traffic flow information and video images using a Hidden Markov Model [8]. Atikom and Pongpaibool, 2006 estimates the road traffic congestion by using vehicle velocity [9]. Krause and Altrock, 1996 uses fuzzy logic to determine six discrete levels of congestion [10]. The system use velocity and vehicle density as inputs its. fuzzy inference system. Sule, 1988; Jia and Li, 2003 uses different factors which affect the road traffic congestion $[11,12]$. This study also uses fuzzy inference systems, but quite different from the previous studies that it examine both manually tuned fuzzy inference systems and adaptive neuro-fuzzy inference system. The motivation behind this study is adaptive neuro-fuzzy and the effectiveness of the manually tuned fuzzy system which de- pends highly on the fuzzy rules and membership function ranges created by human.

There is no systematic way to create these rules and deciding the ranges, and types of membership functions. Therefore, it has to adjust these rules by keeping in mind that congestion is directly proportional to density and inversely proportional to the speed, and adjust ranges and type of the membership function by trial and error method according to the situation until the results are satisfied. The adaptive neuro-fuzzy inference system can solve this problem by automatically creating fuzzy rules according to given inputs and outputs [13-15]. In addition, we limit the traffic congestion status to only five levels-free flow, slow moving, mild congestion, heavy congestion and serious jam, to facilitate a quick and easy to understand report.

\section{Methodology}

To estimate the road traffic congestion of a road segment of Dehradun city, we obtain the traffic flow information at the desired location. The input parameters average speed and density of the vehicle per fixed interval of time are extracted from a video file using manual technique. Subjective congestion evaluation is conducted by watching a traffic video, and the average traffic congestion of

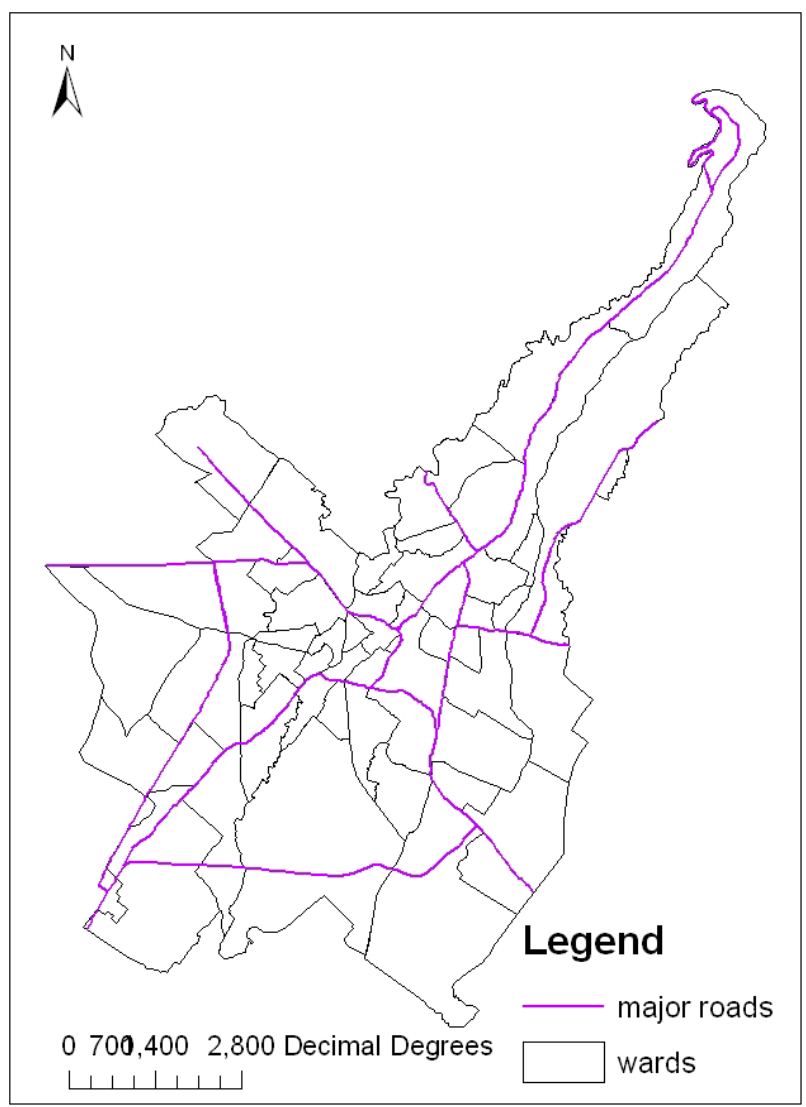

Figure 1. Location of the study area. 
each lane of the road in every time interval is obtained. This information is fed into fuzzy systems, and data sets are prepared in the matrix form containing average speed, density and human evaluated level of congestion. The manually tuned Sugeno-type fuzzy inference system, the manually tuned Mamdani-type fuzzy inference system, and the adaptive neuro-fuzzy inference systems are used to quantify the traffic congestion. These systems are customized with fuzzy logic tools based on the fuzzy set theory. Figure 2 shows the flow chart using three types of fuzzy inference systems.

Fuzzy Inference System: Fuzzy inference systems

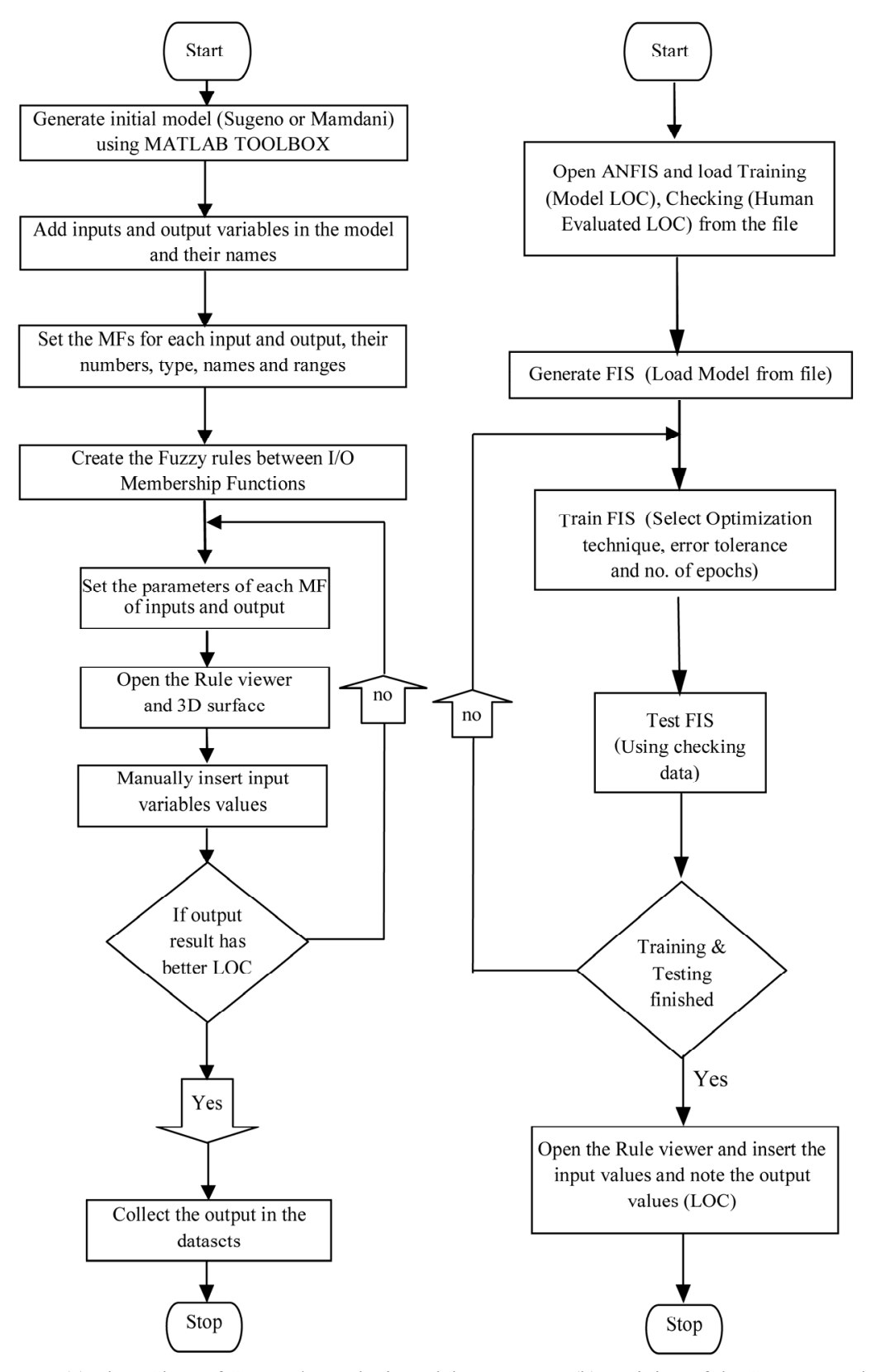

(a) Flow Chart of Sugeno/Mamdani Model

Figure 2. Methodology of fuzzy inference systems. can handle the situations where there are uncertainties are involved, such as problems that depend on the human feelings and expertise. Therefore fuzzy inference systems are suitable for estimating road traffic congestion where different people may feel differently in the same congestion situations. There are two main parts of the fuzzy inference systems 1) input and output membership functions, whose ranges are manually defined by us to fit with input/output logics; and 2) fuzzy rules which are manually designed by a programmer [16]. FISs are successfully applied in the field such as automatic control, data classification, decision analysis, expert systems, and

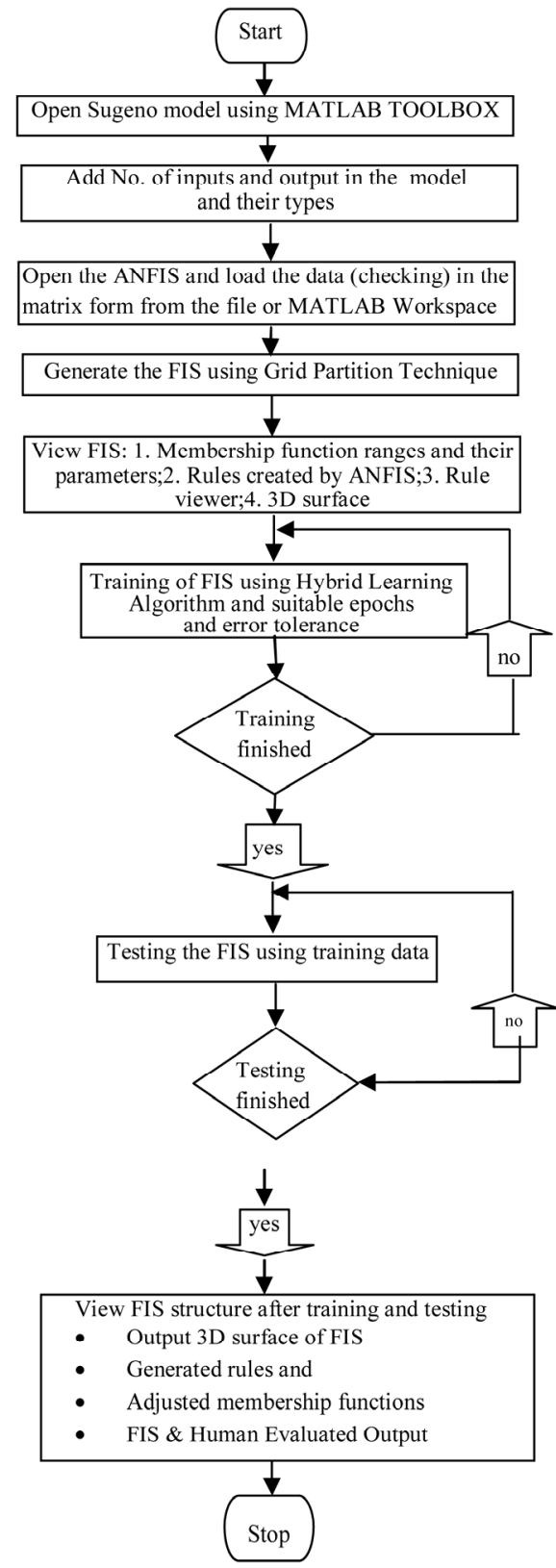

(c) Flow Chart of ANFIS Model 
computer vision. Because of its multidisciplinary nature, FISs are also associated with a number of names, such as fuzzy rule-base systems, fuzzy expert systems, fuzzy modeling, fuzzy associated memory, fuzzy logic controllers, and simply fuzzy systems.

There are mainly six conventional blocks namely input, fuzzification, knowledge base, decision-making unit, defuzzification and output for evaluating the crisp value of output variable in the fuzzy inference system (Figure 3).

Mamdani Model: Mamdani FIS is the most used in the developing fuzzy models. Mamdani architecture used in this paper for estimation of road traffic congestion is illustrated in Figure 4 with two inputs, one output variables and nine fuzzy rules, which consists of five layers of nodes. Out of five layers, first and fourth layers consist of adaptive nodes there are Fuzzification and Defuzzification, and are called Fuzzy layer and De-fuzzy layer, while the second, third and fifth layers consists of fixed nodes there are Rules (or product), Normalization and Summation, and are called product layer, normalization layer and summation layer respectively. The rule base for Mamdani model can be written as

Fuzzy Rule :

$$
\begin{aligned}
& \underbrace{\text { If }\left(x \text { is } A_{i}=M_{1, i}\right) \operatorname{AND}\left(y \text { is } B_{i}=M_{2, i}\right)}_{\text {Premise Part }} \\
& \underbrace{\text { Then } f_{i}=M_{o, j}}_{\text {Consequent Part }}
\end{aligned}
$$

where $x, y, A_{i}$ and $B_{i}$ represent the input1, input2, linguistic label of input1 (slow, medium etc.), and linguistic label of input2 respectively, and $M_{1 i}, M_{2 i}, f_{j}$ and $M_{o j}$ represent the ith MF of input1 (x), the ith MF of input2 $(y)$, the output of the $j$ th rule, and the $j$ th output MF respectively. Both input and output MF have their own parameters depending upon the shape of the MF and are called premise, and consequent parameters respectively.

The computational mechanism of Mamdani FIS at each layer is explained as follows:

Layer 1 (Fuzzification Layer): In this layer the, crisp input values are converted to the fuzzy values sby the input MFs, and the output of every node is the fuzzy membership grade of the inputs, which are given by

$$
\begin{array}{ll}
O_{1, i}=M_{1, i}(x) & \text { for } i=1,2,3 \\
O_{1, i}=M_{2, i}(y) & \text { for } i=1,2,3
\end{array}
$$

where $O_{1, i}$ are the membership grade of a fuzzy set $\{\mathrm{A} 1$, $\mathrm{A} 2, \mathrm{~A} 3, \mathrm{~B} 1, \mathrm{~B} 2, \mathrm{~B} 3\}$. In this paper, the following trapezoidal MFs for the inputs are used

1) Trapezoidal MFs: (see Equation (2))

The another membership functions are also used in this study as input MF.

2) Generalized bell MFs:

$$
M_{k, i}(x)=\operatorname{Gbell}\left(x: a_{k, i}, b_{k, i}, c_{k, i}\right)=\frac{1}{1+\left[\left(\frac{x-c_{k, i}}{a_{k, i}}\right)^{2}\right]^{b_{k, i}}}(3)
$$

where $\left(a_{k i}, b_{k i}, c_{k i}, d_{k i}\right)$ are the parameters of membership functions, known as premise parameters that characterize the shape of the input MFs. Where $k=1,2$ for first and second input respectively.

Layer 2 (Rule Layer): Every node in this layer is a circle node (fixed node) labeled $\pi$, whose output is the product of all incoming inputs and the output represents the firing strength or weighting factor of a fuzzy control rule. The node generates the output by cross multiplying all the incoming inputs and is given by

where

$$
O_{2, j}=W_{j}=M_{1, i}(x) \times M_{2, j}
$$

$$
j= \begin{cases}1,2,3 & \text { for } i=1 \\ 4,5,6 & \text { for } i=2 \\ 7,8,9 & \text { for } i=3\end{cases}
$$

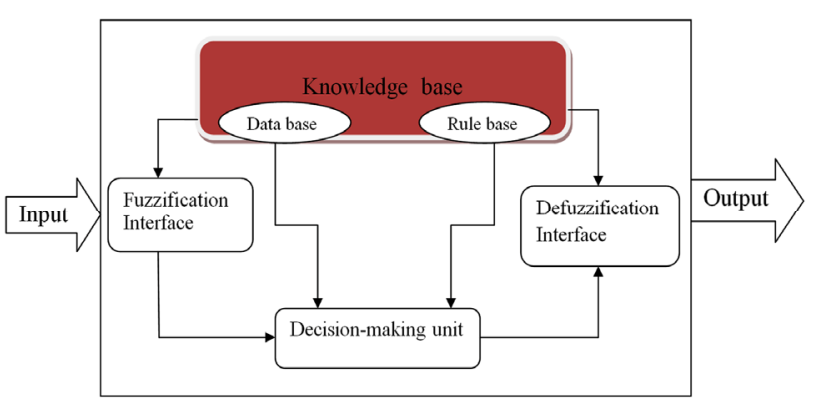

Figure 3. Block diagram of fuzzy inference system.

$$
M_{k, i}(x)=\operatorname{Trap}\left(x: a_{k, i}, b_{k, i}, c_{k, i}, d_{k, i}\right)=\left\{\begin{array}{cc}
0, & x \leq a_{k, i} \\
\frac{x-a_{k, i}}{b_{k, i}-a_{k, i}}, & a_{k, i} \leq x \leq b_{k, i} \\
1, & b_{k, i} \leq x \leq c_{k, i} \\
\frac{d_{k, i}-x}{d_{k, i}-c_{k, i}}, & c_{k, i} \leq x \leq d_{k, i} \\
0, & d_{k, i} \leq x
\end{array}\right.
$$




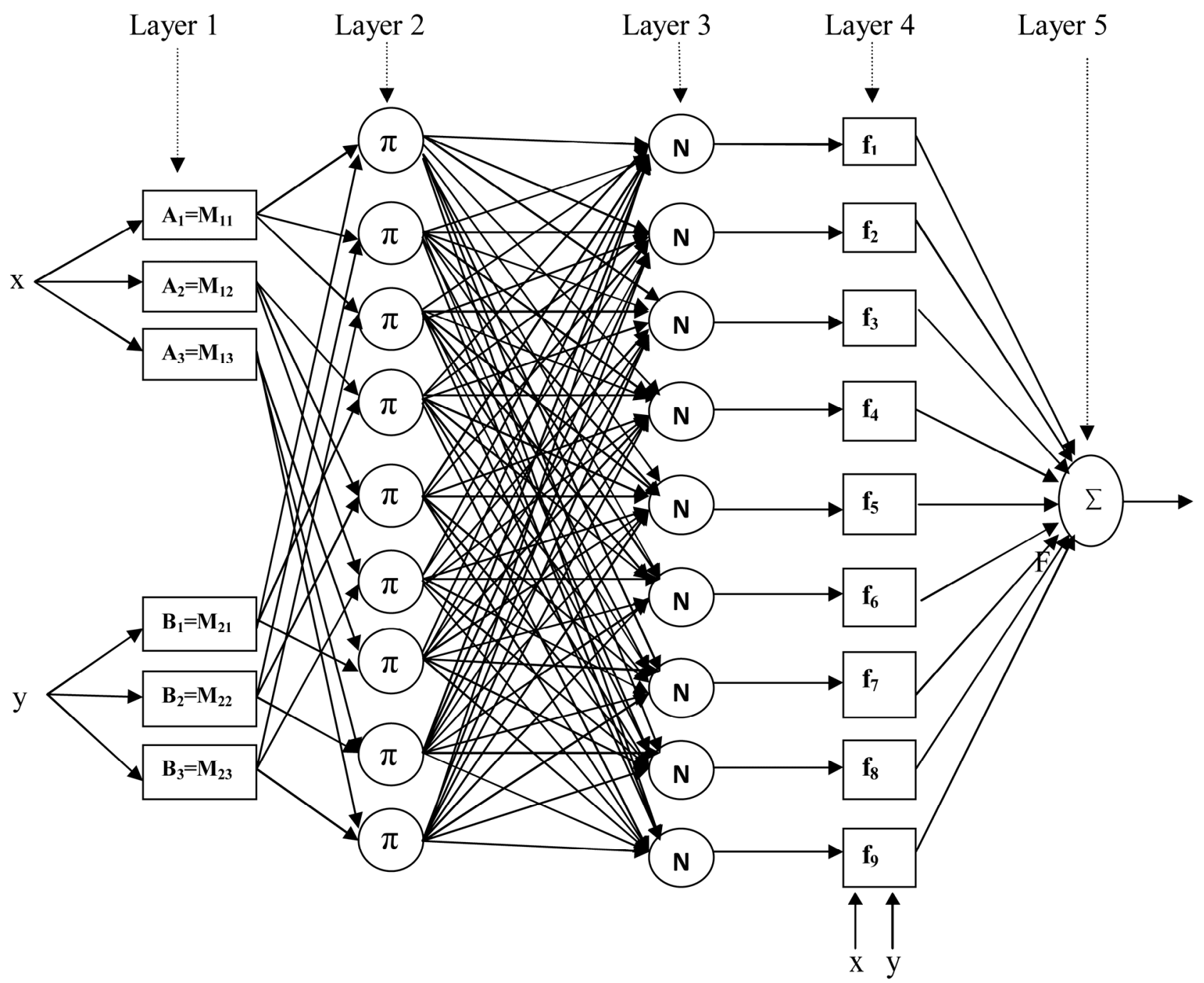

Figure 4. Fuzzy inference system architecture with two inputs, one output and three rules.

Layer 3 (Normalization Layer): Every node of this layer calculates the weight, which is normalized. For convenience, the outputs of this layer are called normalized firing strengths.

$$
O_{3, j}=\overline{W_{j}}=\frac{W_{j}}{\sum_{j=1}^{9} W_{j}} \quad j=1,2, \cdots, 9
$$

where $W_{j}$ is the output of layer 2 .

Layer 4 (Defuzzification Layer): The parameters in this layer are referred to as consequent parameters. The output of every node of this layer is simply the product of the normalized firing strength and a first order polynomial.The output of every node is

$$
O_{4, j}=O_{3, j} \times f_{j}=O_{3, j} \times M_{o, j} \quad j=1,2, \cdots, 9
$$

where $O_{3, j}$ is the output of the layer 3 and $f_{j}$ is the output of the $j$ th fuzzy rule and $M_{\mathrm{oj}}$ is the MFs of $j$ th output. In this paper, MFs for the output are of trapezoidal shape as defined in Equation (2). The another membership func- tions are also used in this study as output MF

1) Linear MFs:

$$
M_{o, j}(x, y)=\operatorname{Linear}\left(x, y: p_{j}, q_{j}, r_{j}\right)=p_{j} x+q_{j} y+r_{j}
$$

2) Constant MF:

$$
M_{o, j}=\text { Cons } \tan t\left(r_{j}\right)=r_{j}
$$

where $p_{j}, q_{j}, r_{j}$ are the parameters of the output MFs corresponding to $j$ th fuzzy rule, known as consequent parameters, characterized by shape of the MFs.

Layer 5 (Summation Layer): The single node in this layer is a circle node (fixed node) labeled $\sum$ which computes the overall output as the summation of all incoming outputs from layer 4 i.e.

$$
\begin{aligned}
O_{5} & =\sum_{j=1}^{9} O_{4, j}=\sum_{j=1}^{9} O_{3, j} \times f_{j}=\frac{\sum_{j=1}^{9} W_{j} \times f_{j}}{\sum_{j=1}^{9} W_{j}} \\
& =\overline{W_{1}} \times f_{1}+\overline{W_{2}} \times f_{2}+\cdots+\overline{W_{9}} \times f_{9}
\end{aligned}
$$


In this study, all the premise and consequent parameters are manually decided in MATLAB tools.

Sugeno Model: The architecture and the fuzzy reasoning of Sugeno model is same as that of the Mamdani model, but it has only linear or constant type of MFs for the output variable. In this study we use the trapezoidal MFs for both the inputs (Speed and Density) and constant MFs for the output (LOC). Here also all the premise and consequent parameters are manually decided in the MATLAB tools.

ANFIS Model: Adaptive Neuro-Fuzzy Inference System (ANFIS) is one of the most successful hybrid modeling technique which combines the adaptive learning capability of Artificial Neural Network (ANN) along with the intuitive Fuzzy logic (FL) into a single capsule. For a given input/output dataset, the ANFIS generates the fuzzy inference system (FIS) using grid partition technique and membership functions parameters are adjusted (tuned) automatically until reach the optimal solution using either a backpropagation algorithm or in combination with least squares type method (hybrid learning method). We use the adaptive neuro-fuzzy inference system (ANFIS) [17], which use the hybrid learning algorithm [13] to create rules and adjust membership function parameters to fit the training data. The membership functions used in ANFIS are gbell's functions for inputs and linear functions for output. We train our ANFIS under 100 epochs.

Depending on the types of fuzzy reasoning and "ifthen" rules, Sugeno's fuzzy model, the output of each rule is a linear combination of input variables plus a constant term or purely constant, because membership function of output variable are only linear or constant type and the final output is the weighted average of each rule's output. Mamdani Model also has same units as in Sugeno model, but only difference is that the membership function of output variable may have different type like trapezoidal, triangulat, Gaussian, exponential etc. [18]. In our case we chose the trapezoidal membership function for the output variable (LOC). The ANFIS is like a fuzzy inference systems, except that here by using a learning algorithm (either a back propagation alone or in combination with a least squares estimation) the parameters of input and output membership function of a fuzzy inference system constructed by ANFIS, have been tuned (adjusted) automatically based on the training data until reach the optimal solution.

\subsection{Data Preparation}

The road traffic video is recorded by a video camera. The road traffic video is 90 minutes long taken in the afternoon (15:07 to 16:37 hours) of February 2, 2011. The speed of the vehicle is calculated by noting the distance between two consecutive poles (in the middle of two lanes i.e. on the divider) that is 24 meters, and also the travel time of a vehicle between these two poles (Figure 5). And thus the average speed and number of vehicles (road density) per 20-second and also per 40-second interval of time is collected in the form of matrix. The average speed and density every 20 -second and 40 -second become the input of our fuzzy inference systems (Sugeno \& Mamdani). Another type of input besides vehicle density and speed, is the human evaluated of congestion level. By watching the traffic video several times, and we form a common sense to decide the level of congestion (LOC) every 20-second and 40-second interval of time. Here we judging D/S (Density over Speed ratio), and evaluate five level of congestion, namely free flow, slow moving, mild congestion, heavy congestion and serious jam, ranging from " 0 " to " 3 ", in which " 0 " means free flow and " 3 " means serious jam. By this way six datasets are prepared in the form of matrices, in which column 1 is average speed, column 2 is density and column 3 is LOC (level of congestion), and each dataset contains 68 data pairs (no. of rows), thus we have six $68 \times 3$ matrix datasets as:

Dataset I: Average speed, density and LOC every 20-second interval of Lane I (left lane);

Dataset II: Average speed, density and LOC every 40-second interval of Lane I (left lane);

Dataset III: Average speed, density and LOC every 20-second interval of Lane II (right lane);

Dataset IV: Average speed, density and LOC every 40-second interval of Lane II (right lane)

Dataset V: Average speed, total density and LOC every 20 -second interval of Lane I \& Lane II;

Dataset VI: Average speed, total density and LOC every 40 -seconds interval of Lane I \& II.

\subsection{Accuracy Assessment}

For each input pair (Speed and Density) there is an output value of LOC which is called a data point, the human evaluated LOC is taken as the standard data of LOC and depends on the human perceptions, and the model evaluated LOC is taken as the validation data and depends upon the adjustment of input/output membership functions of the model. The performance of the model is evaluated by a metric, called accuracy which shows how many output data points of the model match with the corresponding human evaluated output data points. The accuracy of the system is defined by Equation (10)

$$
\text { Accuracy }=\frac{(\text { Total Data Points }- \text { Incorrect Data Points })}{\text { Total Data Points }}(10)
$$

In addition, to measure how far the incorrect data points are from the human opinion, another metric is called average deviation and is given by 

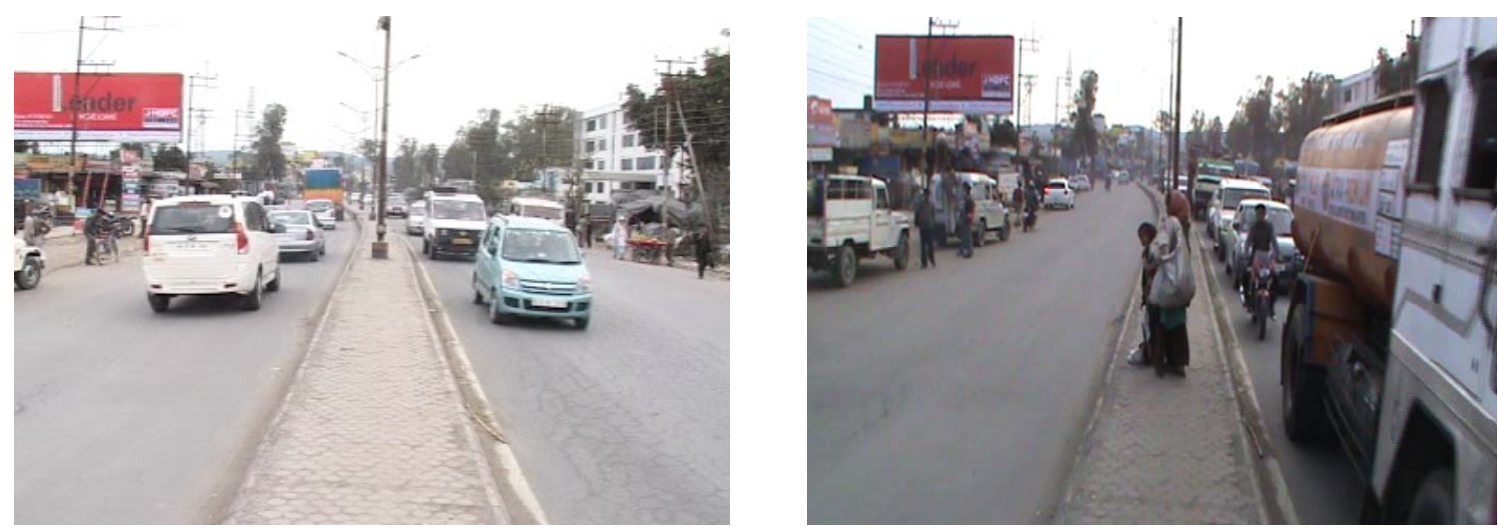

Figure 5. Images taken from traffic video.

Average Deviation

$=\frac{\sum \mid \text { Fuzzy Score }- \text { Human Opinion Score } \mid}{\text { TotalData Points }}$

Human Opinion Score is the LOC rated by human pioneers, corresponding to each pair of inputs, and Fuzzy Score is the LOC rated by the fuzzy system, corresponding to the same input pair. The lower value of average deviation means higher the accuracy of the system and vice-versa.

\section{Results and Discussions}

There are eighteen experiments on LOC evaluation based on the derived parameters described previously, six experiments for each technique. In each experiment, we vary types of input parameters and the evaluation interval. In all the experiments the membership function ranges are changed. There are three membership functions of Speed namely slow, medium and fast, three membership function of Density namely low, medium and high. The MFs of output LOC are different for different inference systems, but the range of the level of congestion $(0-3)$ is same for all the experiments. For each model, the MFs of output (LOC) are nine in numbers namely-2 free flow, 2 slow moving, 1 mild congestion, 2 heavy congestion and 2 serious jam. The "if-then" fuzzy rules are applied on the experiments (Sugeno, 1983), as follows:

Rule 1: If (Speed is slow) AND (Density is high) then (LOC is serious jam1);

Rule 2: If (Speed is slow) AND (Density is medium) then (LOC is serious jam2);

Rule 3: If (Speed is slow) AND (Density is low) then (LOC is heavy congestion 1);

Rule 4: If (speed is medium) AND (Density is high) then (LOC is heavy congestion2);

Rule 5: If (Speed is medium) AND (Density is medium) then (LOC is mild congestion)

Rule 6: If (speed is medium) AND (Density is low) then (LOC is slow moving2);
Rule 7: If (Speed is fast) AND (Density is high) then (LOC is slow moving1);

Rule 8: If (Speed is fast) AND (Density is medium) then (LOC is free flow2);

Rule 9: If (speed is fast) AND (Density is low) then (LOC is free flow1).

Experiment I: Average Speed, Density Evaluation of Lane I, 20-Second by Sugeno Model

Inputs into the Sugeno model are the average speed and density in the left lane. Here the input MFs are trapezoidal type and the output MFs are constant type. The range of the speed is between $0-60 \mathrm{~km} / \mathrm{hr}$ and density range between $0-40$ vehicles per 20 seconds. The MF ranges for average speed are less than $18 \mathrm{~km} / \mathrm{hr}$ for slow, $15-35 \mathrm{~km} / \mathrm{hr}$ for medium and more than $30 \mathrm{~km} / \mathrm{hr}$ for fast. The MF ranges for density are 0-10 vehicles for low, 7 - 22 vehicles for medium and more than 18 vehicles for high. The output LOC has nine MFs, namely 2 free flow, 2 slow moving, 1 mild congestion, 2 heavy congestion and 2 serious jam corresponding to the ranges $0,0.67,1,1.33,1.67,2,2.33,2.67$, and 3 . First we get the LOC by manually putting the values of speed and density in the model and then train this model using ANFIS tool using model output as training data and under 80 epochs. ANFIS automatically adjusted MF parameters and gives better result. The MFs and Fuzzy rules, and 3D surface are shown in Figure 6.

The list of output LOC for every data pair is shown in the Table 1. The human evaluated values and model based LOC values are closely matching with respected to the given input variables. The difference of 0.20 in values of LOC is considered as acceptable error for convenience $(0.20$ is about $7 \%$ of LOC range). The MFs above yield the accuracy of $55.88 \%$, and average deviation of 0.0426323 levels.

Experiment II: Average Speed and Density Evaluation of Lane II, 20-Second by Sugeno Model

In a 20 second evaluation interval and Sugeno-type inference system, inputs fed into the system i.e. average speed and density of the right lane. The model is trained 


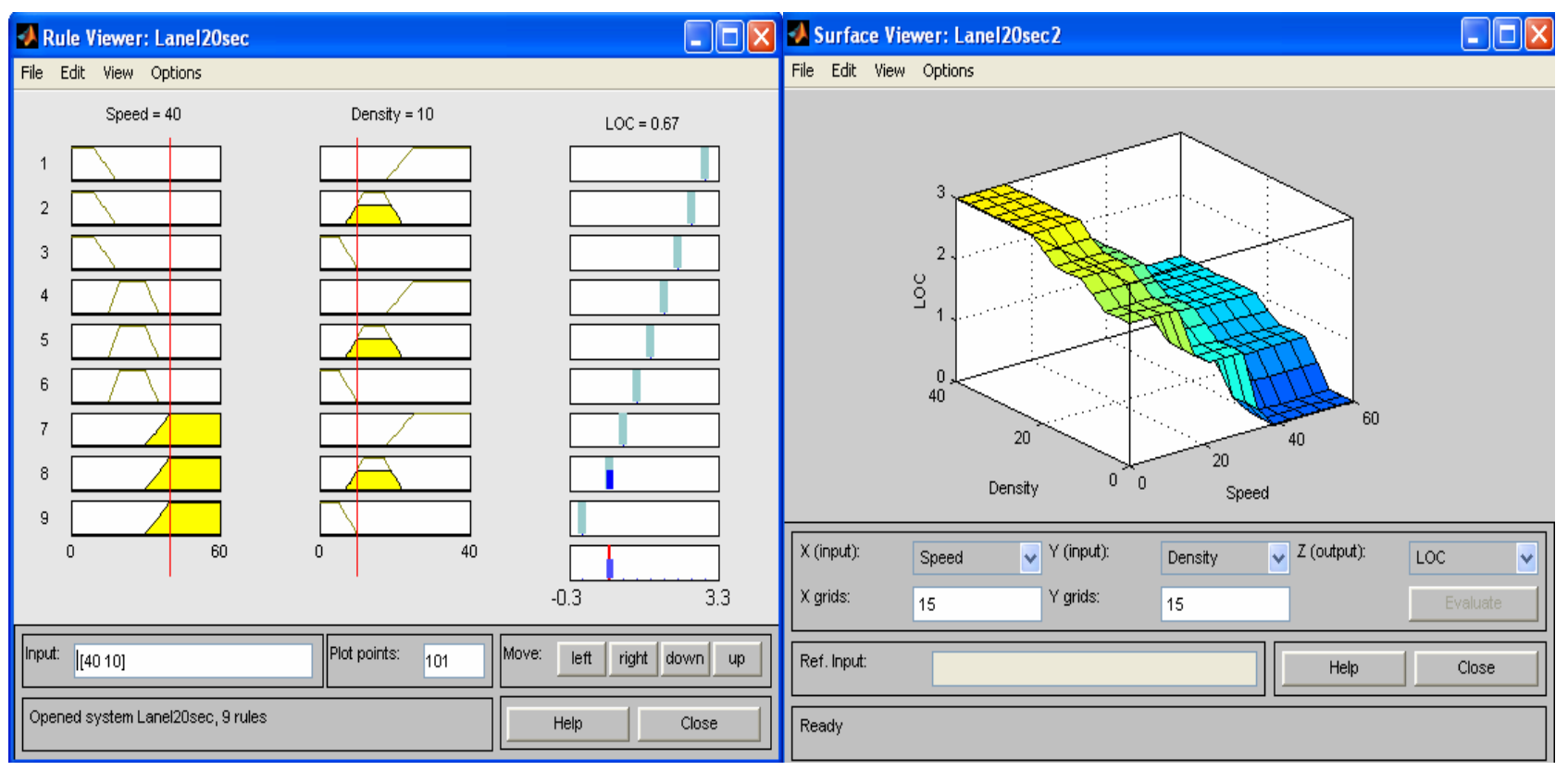

Figure 6. MATLAB program showing the MFs and fuzzy rule, and 3D surface of the model.

Table 1. Comparative table of human evaluated vs model Loc.

\begin{tabular}{cccccc}
\hline $\begin{array}{c}\text { Average Speed } \\
(\mathbf{k m} / \mathbf{h r})\end{array}$ & $\begin{array}{c}\text { Density } \\
\text { (No. of vehicles) }\end{array}$ & $\begin{array}{c}\text { LOC } \\
\text { (Density/Speed)*3 }\end{array}$ & $\begin{array}{c}\text { Human Opinion } \\
\text { LOC }\end{array}$ & $\begin{array}{c}\text { Model } \\
\text { LOC }\end{array}$ & Time \\
\hline 40 & 10 & 0.45 & 0.45 & 0.67 & $15: 07: 07$ \\
25 & 7 & 0.84 & 1.1 & 1.33 & $15: 09: 13$ \\
28 & 4 & 0.43 & 1.1 & 1.33 & $15: 11: 52$ \\
12 & 10 & 2.50 & 2.85 & $* 2.67$ & $15: 15: 00$ \\
16 & 14 & 2.68 & 2.1 & $* 2.2$ & $15: 15: 40$ \\
$\cdots$ & $\cdots$ & $\cdots$ & $\cdots$ & $\cdots$ & $\cdots$ \\
$\cdots$ & $\cdots$ & $\cdots$ & $\cdots$ & $\cdots$ & $\cdots$ \\
\hline
\end{tabular}

by ANFIS under same conditions as in experiment I. The above MFs yield the accuracy of $70.68 \%$ and average deviation of 0.0018382 levels.

Experiment III: Average Speed and Density Evaluation of Lane I, 40-Second by Sugeno Model

Similarly, a 40-second evaluation interval and Sugenotype inference system is tested with average speed and density of left lane. The range of the speed is between 0 $60 \mathrm{~km} / \mathrm{hr}$ and density range between $0-60$ vehicles per 40 seconds, and the range of LOC is $0-3$.

The ranges of the MFs of density are $0-20$ vehicles for low, 15 - 35 vehicles for medium and more than 30 vehicles for high, and the average speed and output MF ranges are same as in experiment $\mathrm{I}$. The model is trained by ANFIS under same conditions as in experiment I. The MFs above yield the accuracy of $57.35 \%$, and average deviation of 0.0600294 levels. This performance is better than that of experiment I, which leads us to think that the evaluation interval may affect the performance of the fuzzy inference system.

\section{Experiment IV: Average Speed and Density Evalua-} tion of Lane II, 40-Second by Sugeno Model

Here, 40-second evaluation interval is given into the system along with average speed and density of the right lane. All inputs, outputs, fuzzy rules, and MFs (shape, and names) are the same as those in experiment I, and ranges and training parameters are same as in experiment III. Here we again want to see the effect of evaluation interval on level of congestion.

The MFs yield the accuracy of $55.88 \%$, and the average deviation of 0.0634117 levels. This performance is not better than that of experiment II, which leads us to think that, it is not necessary that the evaluation interval may affect the performance of the fuzzy systems.

Experiment V: Average Speed and Total Density Evaluation of Lane I \& II, 20-Second by Sugeno Model

In this experiment, evaluation interval is reduced to 20 -second, and inputs into the model are the average speed and total density of both the lanes. The MFs yield the accuracy of $67.65 \%$, and the average deviation of 


\subsection{0 levels.}

Experiment VI: Average Speed and Total Density Evaluation of Lane I \& II, 40-Second by Sugeno Model

In this experiment, we assume 40 second evaluation interval with inputs average speed and total density of both the lanes into the system. All inputs, outputs, fuzzy rules and MFS (shape, and names) are the same as those in experiment I. Average speed range in the experiment is same as experiment $\mathrm{I}$, and we have changed the range of total density to $0-80$. The MF ranges of total density are low (below 22 vehicles), medium (18 - 40 vehicles) and high (above 35 vehicles). The MFs yield the accuracy of $72.05 \%$, and the average deviation of 0.1564117 levels. Here, the evaluation interval improves the accuracy of the model, it means as evaluation interval increases, accuracy of the model also increases and viceversa.

Experiment VII: Average Speed and Density Evaluation of Lane I, 20-Second by Mamdani Model

In this experiment, Mamdani-type inference system is used with 20-second evaluation interval. Inputs into the system are the average speed and density of left lane. Here the input MFs are trapezoidal type and the output MFs are also trapezoidal. The range of the speed is between $0-60 \mathrm{~km} / \mathrm{hr}$ and density range is between $0-40$ vehicles per 20 -seconds. The MF ranges for average speed are less than $18 \mathrm{~km} / \mathrm{hr}$ for slow, $15-35 \mathrm{~km} / \mathrm{hr}$ for medium and more than $30 \mathrm{~km} / \mathrm{hr}$ for fast. The MF ranges for density are 0 - 10 vehicles for low, $8-22$ vehicles for medium and more than 19 vehicles for high. The output level of congestion has nine members, namely 2 free flow, 2 slow moving, 1 mild congestion, 2 heavy congestion and 2 serious jam corresponding to the ranges below 0.65 , between $0.55-1.25,1.15-1.85,1.75-2.45$ and above 2.35 . The LOC is obtained by manually putting the values of speed and density in the model (in rule viewer) and then compare with the human evaluated LOC. In Figure 7, shows the MFs of input/output variable and Fuzzy Rule viewer, and 3D surface of the model. The MFs above yield the accuracy of $82.35 \%$, and the average deviation of 0.1115213 levels.

Experiment VIII: Average Speed and Density Evaluation of Lane II, 20-Second by Mamdani Model

In this experiment, Mamdani-type inference system is used with 20 -second evaluation interval, and inputs into the system are the average speed and density in the right lane. All inputs, output, fuzzy rules, and MFs (shape, ranges and names) are the same as those in experiment VII. The MFs above yield the accuracy of $83.82 \%$, and the average deviation of 0.0917643 levels.

Experiment IX: Average Speed and Density Evaluation of Lane I, 40-Second by Mamdani Model

In this experiment, a 40 -second evaluation interval, and inputs into the system are the average speed and density in the left lane. All inputs, output, fuzzy rules, and MFs (shape, and names) are the same as those in experiment VII. The ranges of input speed and output LOC and their MFs are same as those of experiment VII. The range of density between 0 - 60 vehicles per 40 seconds and the ranges of MFs of density are, below 20 for Low, between 15 - 35 for Medium and above 31 for High. The MFs above yield the accuracy of $77.94 \%$, and the average deviation of 0.1112561 levels. This performance is not better than that of experiment VII, which infers that it is not necessary that the evaluation interval could improve the accuracy of the Mamdani-type fuzzy inference system.
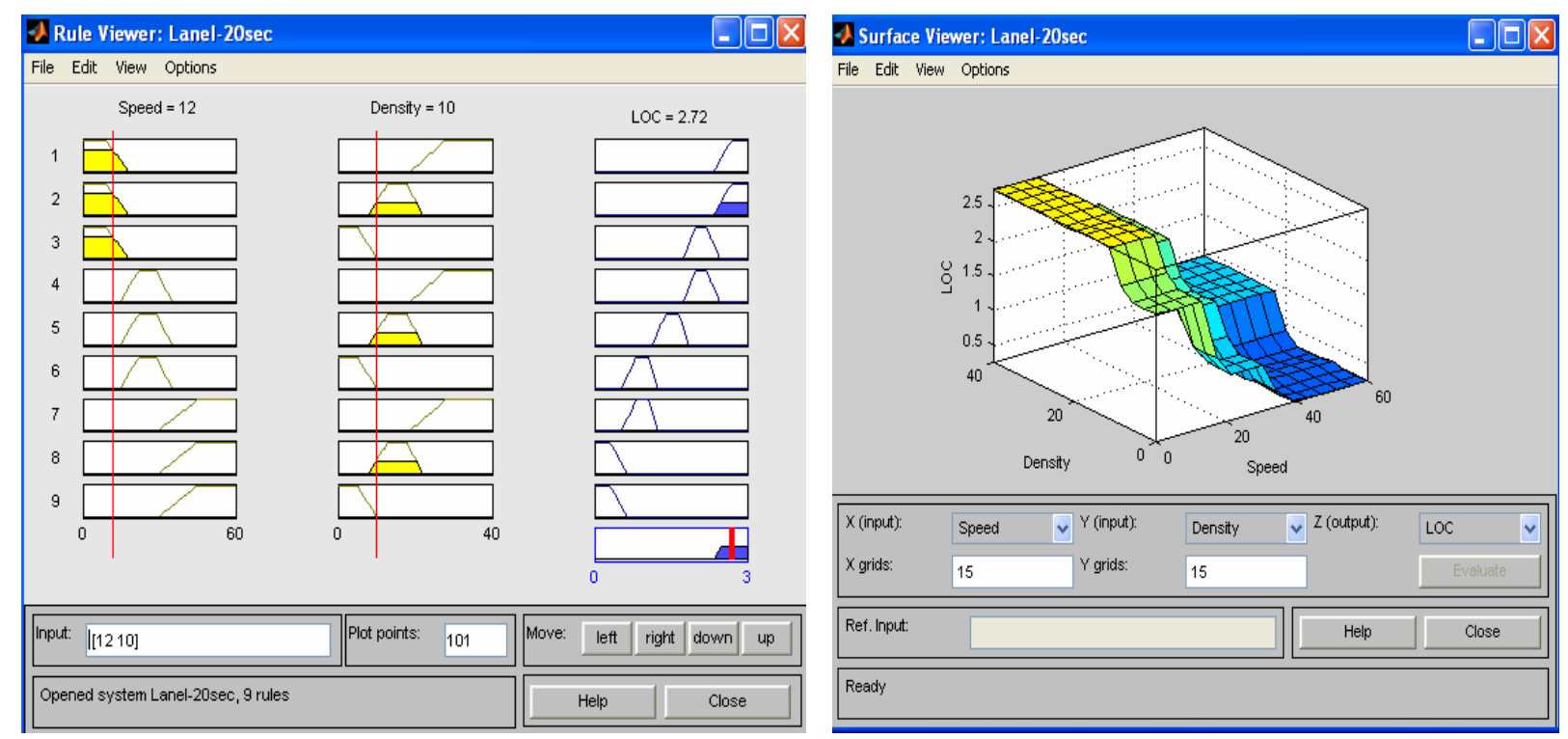

Figure 7. MATLAB program showing MFs and fuzzy rules, and 3D surface of the model. 
Experiment X: Average Speed and Density Evaluation of Lane II, 40-Second by Mamdani Model

In this experiment, under similar conditions of experiment IX, the MFs yield the accuracy of $73.52 \%$, and the average deviation of 0.1441361 levels. This performance is not better than that of experiment VIII, which again leads to think that it is not necessary that the evaluation interval could improve the accuracy of the Mamdani-type fuzzy inference system.

Experiment XI: Average Speed and Density Evaluation of Lane I \& II, 20-Second by Mamdani Model

In this experiment, Mamdani-type inference system is used with 20 -second evaluation interval, and inputs into the system are the average speed and density of both the lanes. All inputs, output, fuzzy rules, and MFs (shape, and names) are the same as those in experiment VII. The ranges of inputs and output LOC and their MFs of are same as those of experiment IX. The MFs above yield the accuracy of $64.70 \%$, and the average deviation of 0.1756654 levels

Experiment XII: Average Speed and Density Evaluation of Lane I \& II, 40-Second by Mamdani Model

This experiment is deployed with Mamdani-type inference system with 40 -second evaluation interval, and inputs into the system are the average speed and density of both the lanes. All inputs, output, fuzzy rules, and MFS (shape, and names) are the same as those in experiment VII. The ranges of input speed and output LOC and their MFs are same as those in experiment VII. The range of density between 0 - 60 vehicles per 40 seconds and the ranges of membership functions of density are, below 22 for Low, between 18 - 40 for Medium and above 35 for High. The MFs above yield the accuracy of $63.23 \%$, and the average deviation of 0.1867251 levels. This performance is not much better after increasing the evaluation interval for both the lanes. Again it is not clear that evaluation interval could improve the performance of the model.

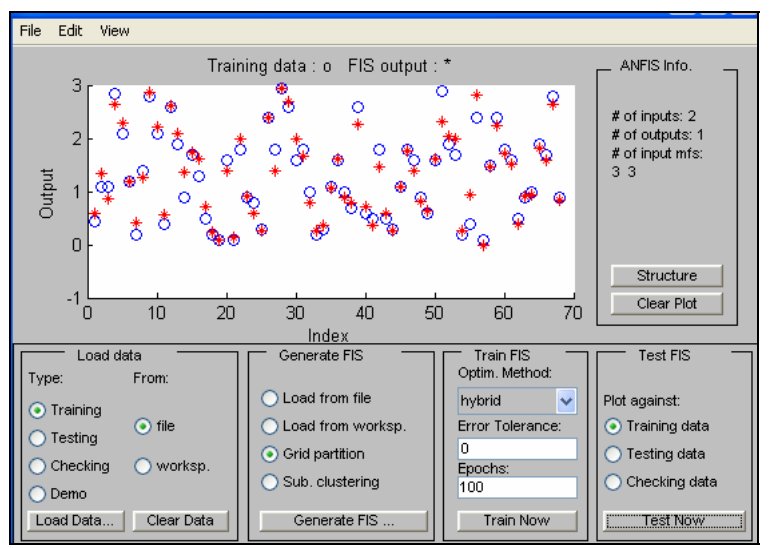

(a)
Experiment XIII: Average Speed and Density Evaluation of Lane I, 20-Second by ANFIS Model

ANFIS model is deployed with the three gbell's MF for each input, and nine linear MF for the output. The ranges of each variable are automatically decided by using grid Partition technique using Matlab ANFIS tools. Inputs into the system are the inputs average speed, density and output human opinion LOC of left lane in the form of a matrix (dat.file). The input-output dataset are then passed through the ANFIS tool for learning and automatically adjusting the output. After 100 epochs, and using hybrid learning algorithm, the automatically generated FIS is trained, and thus the ANFIS is ready to evaluate the congestion status. The ANFIS outputs after training,model and structure are shown in Figure 8(a) and (b). The MFs above yields the accuracy of $72.05 \%$, and the average deviation of 0.0005667 levels.

Experiment XIV: Average Speed and Density Evaluation of Lane II, 20-Second by ANFIS Model

In this experiment, we repeat the same procedure as in experiment XIII, but the input-output dataset of left lane is loaded into the ANFIS tool.

The MFs above yield the accuracy of $80.88 \%$, and the average deviation of 0.0870766 levels.

Experiment XV: Average Speed and Density Evaluation of Lane I, 40-Second by ANFIS Model

In this experiment, we repeat the same procedure as in experiment XIII, but the evaluation interval increases from 20 seconds to 40 seconds, and input-output dataset of left lane is loaded into the ANFIS tool. The MFs above yields the accuracy of $88.23 \%$, and the average deviation of 0.0109558 levels. This performance is better than that of experiment VII, which leads to think that the evaluation interval may affect the performance of the ANFIS.

Experiment XVI: Average Speed and Density Evaluation of Lane II, 40-Second by ANFIS Model

In this experiment, we repeat the same procedure as in

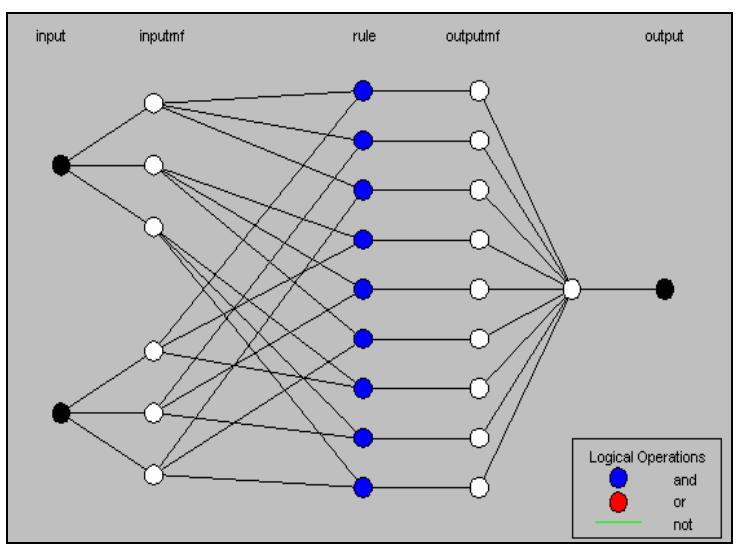

(b)

Figure 8. (a) Training and FIS output; (b) ANFIS model structure. 
experiment $\mathrm{XV}$, but input-output dataset of right lane is loaded into the ANFIS tool. The MFs yield the accuracy of $77.94 \%$, and the average deviation of 0.0018514 levels. This performance is impressive than that of experiment XIV, which leads to think that it is not necessary that the evaluation interval could improve the accuracy of the ANFIS.

Experiment XVII: Average Speed and Total Density Evaluation of Lane I \& II, 20-Second by ANFIS Model

In this experiment, we repeat the same procedure as in experiment XIII, but the input-output dataset of both the lanes is loaded into the ANFIS tool. The MFs yield the accuracy of $73.52 \%$, and the average deviation of 0.0018 661 levels.

Experiment XVIII: Average Speed and Total Density Evaluation of Lane I \& II, 40-Second by ANFIS Model

In this experiment, the same process is repeated as in experiment XIII and XVII, but the evaluation interval increases from 20 -second to 40 -second. The MFs above yield the accuracy of $72.05 \%$, and the average deviation reach to 0.0003088 levels. This performance is not again very impressive after increasing the evaluation interval. Therefore, it is not conclusive that evaluation interval could improve the performance of ANFIS. Table 2 summarizes the accuracy and average deviation of all the above experiments.

In most of the cases the accuracy achieved by ANFIS model is higher than the manually tuned Mamdani and Sugeno model. However, the accuracy achieved by manually tuned Mamdani and Sugeno model is very near to that of ANFIS model, which shows that fuzzy inference systems can capture the human perceptions as well. Overall the maximum accuracy achieved by Sugeno model is $72.05 \%$, Mamdani model is $83.82 \%$ and ANFIS model is $88.23 \%$. These models are also applied after changing the evaluation interval of each lane individually and combination of both and evaluate the accuracy of the models. For left lane, as the evaluation interval increases from 20 seconds to 40 seconds then the accuracy of Sugeno model increases from $55.88 \%$ to $57.35 \%$, but the same time accuracy of Mamdani model reduces from $82.35 \%$ to $77.94 \%$ and the accuracy of ANFIS model increases from $72.05 \%$ to $88.23 \%$, which shows that it is not necessary that evaluation interval could improve the accuracy of the model. Also for right lane as the evaluation interval increases from 20 seconds to 40 seconds, the

Table 2. Summary of model performance.

\begin{tabular}{|c|c|c|c|c|c|}
\hline Experiment No. & Fuzzy System & Input parameters & Evaluation Interval & Accuracy & Average deviation \\
\hline I & Sugeno-type & Avg.Speed, Density of left lane & 20 seconds & $55.88 \%$ & 0.0426323 \\
\hline II & Sugeno-type & Avg.Speed, Density of right lane & 20 seconds & $70.58 \%$ & 0.0018382 \\
\hline III & Sugeno-type & Avg.Speed, Density of left lane & 40 seconds & $57.35 \%$ & 0.0600294 \\
\hline IV & Sugeno-type & Avg.Speed, Density of right lane & 40 seconds & $55.88 \%$ & 0.0634117 \\
\hline $\mathrm{V}$ & Sugeno-type & Avg.Speed, Total Density of both lanes & 20 seconds & $67.65 \%$ & 0.1573970 \\
\hline VI & Sugeno-type & Avg.Speed, Total Density of both lanes & 40 seconds & $72.05 \%$ & 0.1564117 \\
\hline VII & Mamdani-type & Avg.Speed, Density of left lane & 20 seconds & $82.35 \%$ & 0.1115213 \\
\hline VIII & Mamdani-type & Avg.Speed, Density of right lane & 20 seconds & $83.82 \%$ & 0.0917643 \\
\hline IX & Mamdani-type & Avg.Speed, Density of left lane & 40 seconds & $77.94 \%$ & 0.1112561 \\
\hline $\mathrm{X}$ & Mamdani-type & Avg.Speed, Density of right lane & 40 seconds & $73.52 \%$ & 0.1441361 \\
\hline $\mathrm{XI}$ & Mamdani-type & Avg.Speed, Total Density of both lanes & 20 seconds & $64.70 \%$ & 0.1756654 \\
\hline XII & Mamdani-type & Avg.Speed, Total Density of both lanes & 40 seconds & $63.23 \%$ & 0.1867251 \\
\hline XIII & ANFIS & $\begin{array}{l}\text { Avg.Speed, Density and Human opinion LOC of } \\
\text { left lane }\end{array}$ & 20 seconds & $72.05 \%$ & 0.0005667 \\
\hline XIV & ANFIS & $\begin{array}{l}\text { Avg.Speed, Density and Human opinion LOC of } \\
\text { right lane }\end{array}$ & 20 seconds & $80.88 \%$ & 0.0870766 \\
\hline XV & ANFIS & $\begin{array}{l}\text { Avg.Speed, Density and Human opinion LOC of } \\
\text { left lane }\end{array}$ & 40 seconds & $88.23 \%$ & 0.0109558 \\
\hline XVI & ANFIS & $\begin{array}{l}\text { Avg.Speed, Density and Human opinion LOC of } \\
\text { right lane }\end{array}$ & 40 seconds & $77.94 \%$ & 0.0018514 \\
\hline XVII & ANFIS & $\begin{array}{l}\text { Avg.Speed, Total Density and Human opinion } \\
\text { LOC of both lanes }\end{array}$ & 20 seconds & $73.52 \%$ & 0.0018661 \\
\hline XVIII & ANFIS & $\begin{array}{l}\text { Avg.Speed, Total Density and Human opinion } \\
\text { LOC of both lanes }\end{array}$ & 40 seconds & $72.05 \%$ & 0.0003088 \\
\hline
\end{tabular}


accuracy of Sugeno model reduced from $70.58 \%$ to $55.88 \%$, accuracy of Mamdani model decreases from $83.82 \%$ to $73.52 \%$, and that of ANFIS model decreases from $80.88 \%$ to $77.94 \%$. Similarly for the combination of both the lanes, the accuracy of Sugeno model increases from $67.65 \%$ to $72.05 \%$, accuracy of Mamdani model decreases from $64.70 \%$ to $63.23 \%$, and that of the ANFIS model decreases from $73.52 \%$ to $72.05 \%$, which again shows that it is not necessary that evaluation interval could improve the performance of the model.

\section{Conclusions}

In this study, we proposed the advantage of fuzzy inference system (Sugeno-type, Mamdani-type and adaptive neuro-fuzzy inference system) technology, to evaluate the level of road traffic congestion. Basically the level of congestion (LOC) of road traffics is a continuous variable to express the situation from free flow to serious traffic jam. The performance of our proposed systems is evaluated by measuring accuracy of outputs against human opinion. Through different experiments, we find that the manually tuned fuzzy inference system achieve the accuracy which is very near to the accuracy achieved by adaptive neuro-fuzzy inference system. It means the fuzzy inference system can capture the human expertise better than manual adjustment of input/output membership functions. Two types of fuzzy logic inputs- average speed and density within an interval. We investigate the effect of using single lane traffic information as opposed to two lane information. It is also observed that how the evaluation interval affects the accuracy of the system. The results are not conclusive whether longer interval can improve accuracy, but this effect depends on nature of traffic flow at particular road segment and particular time. The systems used in this study have advantage of minimum requirement of input data, and better accuracy and reduced error margin. Therefore, it is possible to use fuzzy system to evaluate the road traffic congestion with greater accuracy and low error margins. However, accuracy of fuzzy systems depends highly on the types of rules, and how the rules are defined along with membership function ranges.

Future Scope: In general, the congestion is the restriction in the movement of the vehicles on the roads, therefore it can be compared with the impedance of the roads which can be used in the network analysis in GIS. In addition to that, congestion can be evaluated for different times and thus temporal impedance can be calculated which can be used in temporal network analysis.

\section{REFERENCES}

[1] Bureau of Public Roads, "Highway Capacity Manual: Practical applications of Research," US Department of
Commerce, Washington DC, 1950.

[2] D. Branston, "Link Capacity Functions: A Review" Transportation Research, Vol. 10, No. 4, 1976, pp. 223-236. doi:10.1016/0041-1647(76)90055-1

[3] P. Posawang, S. Phosaard, W. Polnigongit and W. PattaraAtikom, "Perception-Based Road Traffic Congestion Classification Using Neural Networks," Proceedings of the World Congress on Engineering, London, 1-3 July 2009.

[4] A. P. Addepalli, "Study of Mixed Traffic Flow Characteristics: A Microscopic Simulation Approachm," M.Tech Thesis, IIT, Madras, 2000.

[5] C.-C. Lee, "Fuzzy Logic in Control Systems: Fuzzy Logic Controller I," IEEE Transactions on Systems, Man and Cybernetics, Vol. 20, No. 2, 1990, pp. 404-418. doi: $10.1109 / 21.52551$

[6] C.-C. Lee, "Fuzzy Logic in Control Systems: Fuzzy Logic Controller II," IEEE Transactions on Systems, Man and Cybernetics, Vol. 20, No. 2, 1990, pp. 419-435. doi: $10.1109 / 21.52552$

[7] A. Kablan, "Adaptive Neuro-Fuzzy Inference System for Financial Trading Using Intraday Seasonality Observation Model," World Academy of Science, Engineering and Technology, Vol. 58, No., 2009, pp. 479-488.

[8] F. Porikli and X. Li, "Traffic Congestion Estimation Using HMM Models without Vehicle Tracking," IEEE Intelligent Vehicles Symposium, Parma, 14-17 June 2004, pp. 188-193. doi:10.1109/IVS.2004.1336379

[9] W. Pattara-Atikom and P. Pongpaibool, "Estimating Road Traffic Congestion Using Vehicle Velocity," Proceeding of 6th International Conference on Telecommunications, Chengdu, June 2006, pp. 1001-1004.

[10] B. Krause and C. von Altrock, "Intelligent Highway by Fuzzy Logic: Congestion Detection and Traffic Control on Multi-Lane Roads with Variable Road Signs," 5th International Conference on Fuzzy Systems, New Orleans, 8-11 September 1996, pp. 1832-1837. doi:10.1109/FUZZY.1996.552649

[11] A. S. Alfa, "Understanding Urban Traffic Congestion during Peak Periods," Proceedings of International Conference on Road and Road Transport Problems (ICORT-88), Roorkee, 12-15 December 1988, pp. 518-527.

[12] L. Jia and C. Li, "Congestion Evaluation from Traffic Flow Information Based on Fuzzy Logic," IEEE Intelligent Transportation Systems, Vol. 1, 2003, pp. 50-53.

[13] P. Mitra, “ANFIS Based Automatic Voltage Regulator with Hybrid Learning Algorithm," International Journal of Advances in Soft Computing and Applications, Vol. 2, 2010.

[14] S. M. Seyedhoseini, "Application of Adaptive Neuro-Fuzzy Inference System in Measurement of Supply Chain Agility: Real Case Study of a Manufacturing Company," African Journal of Business Management, Vol. 4, No. 1, 2010, pp. 83-96.

[15] T. O. S. Hanafy, "A Modified Algorithm to Model Highly Nonlinear System," Journal of American Science, Vol. 6, No. 12, 2010, pp. 747-759.

[16] T. Takagi and M. Sugeno, "Derivation of Fuzzy Control Rules from Human Operator's Control Actions," Pro- 
ceedings of IFAC Symposium on Fuzzy Information, Knowledge Representation and Decision Analysis, Marseilles, 19-21 July 1983, pp. 55-60.

[17] A. P. Paplinski, "Adaptive Neuro-Fuzzy Inference System (ANFIS),” 20 May 2005.

[18] L. A. Zadeh, "Outline of a New Approach to the Analysis of Complex Systems and Decision Processes," IEEE
Transactions on Systems, Man and Cybernetics, Vol. 3 , No. 1, 1973, pp. 28-44.

[19] N. Patchanee, P. Tangamchit and P. Pongpaibool, "Road Traffic Estimation from a GPS-Equipped Car Using Fuzzy Logic," Proceeding of 29th Electrical Engineering Conference, Chonburi, 9-10 November 2006, pp. 1081-1084. 